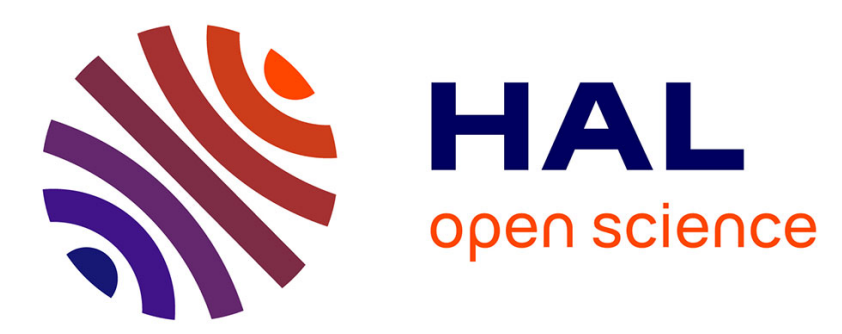

\title{
Advanced Resource Allocation Technique for a Fair Downlink Non-Orthogonal Multiple Access System
}

Marie-Rita Hojeij, Charbel Abdel Nour, Joumana Farah, Catherine Douillard

\section{To cite this version:}

Marie-Rita Hojeij, Charbel Abdel Nour, Joumana Farah, Catherine Douillard. Advanced Resource Allocation Technique for a Fair Downlink Non-Orthogonal Multiple Access System. ICT 2018: 25th International Conference on Telecommunications, Jun 2018, Saint Malo, France. 10.1109/ICT.2018.8464920 . hal-01868945

\section{HAL Id: hal-01868945 \\ https://imt-atlantique.hal.science/hal-01868945}

Submitted on 6 Sep 2018

HAL is a multi-disciplinary open access archive for the deposit and dissemination of scientific research documents, whether they are published or not. The documents may come from teaching and research institutions in France or abroad, or from public or private research centers.
L'archive ouverte pluridisciplinaire HAL, est destinée au dépôt et à la diffusion de documents scientifiques de niveau recherche, publiés ou non, émanant des établissements d'enseignement et de recherche français ou étrangers, des laboratoires publics ou privés. 


\title{
Advanced Resource Allocation Technique for a Fair Downlink Non-Orthogonal Multiple Access System
}

\author{
Marie-Rita Hojeij, Student Member, IEEE, Charbel Abdel Nour, Member, IEEE, Joumana Farah, Member, IEEE, \\ and Catherine Douillard, Senior Member, IEEE
}

\begin{abstract}
In this paper, a novel resource allocation technique is proposed for a power-domain non-orthogonal multiple access scheme using successive interference cancellation in a downlink cellular system. It aims at providing a flexible balancing between throughput and fairness maximization and incorporates unequal power repartition among allocated subbands using waterfilling. Its main strength is the ability to achieve a high level of fairness in every scheduling slot, therefore improving the quality of experience for all users. Fairness is achieved by the introduction of a new efficient scheduling metric within the allocation process. Extensive simulation results show that the proposed metric enhances both long-term and short-term fairness, and improves system capacity, when compared to the conventional proportional fairness scheduler.
\end{abstract}

Index Terms-Non-orthogonal multiple access, power allocation, scheduling, proportional fairness, long-term and short-term fairness.

\section{INTRODUCTION}

Recent radio access technologies are characterized by multiple access schemes which provide the mean for multiple users to access and share resources at the same time. In the 3.9 and $4^{\text {th }}$ generations of mobile communication systems, such as Long-Term Evolution (LTE) and LTE-Advanced, Orthogonal Multiple Access (OMA) based on OFDM (Orthogonal Frequency Division Multiplexing) and Single Carrier Frequency Division Multiple Access (SC-FDMA) were adopted, respectively for the downlink and uplink transmissions. OMA techniques have gained their success from their ability to achieve good system-level throughput performance in packet-domain services and their viability in practical implementations with single-user detection.

However, with the proliferation of smart and machine-tomachine devices, it is expected that the mobile traffic volume supported by future communication networks will be, by 2020 , almost 10 times larger than today's [1]. To respond favorably to such constraints while keeping a high level of user Quality of Experience (QoE), system capacity and user fairness should be largely improved for the future $5^{\text {th }}$ generation $(5 \mathrm{G})$ of mobile communication systems. To this end, Non-Orthogonal Multiple Access (NOMA) has recently emerged as a promising candidate for future radio access. By exploiting an additional

M. Hojeij, C. Abdel Nour and C. Douillard are with IMT Atlantique, Lab-STICC, CNRS UMR 6285, CS 83 818-29238 Brest Cedex 3, France (marie.hojeij, charbel.abdelnour, catherine.douillard@imt-atlantique.fr) an M. Hojeij is also with the Department of Telecommunications, Faculty of Engineering, Holy-Spirit University of Kaslik, P.O. Box 446, Jounieh, Lebanon. J. Farah is with the Department of Electricity and Electronics, Faculty of Engineering, Lebanese University, Roumieh, Lebanon (joumana.farah@ul.edu.lb). multiplexing domain, Power Domain NOMA (PD-NOMA) allows the cohabitation of two or more users per subband at the transmitter side on top of the OFDM layer. Multi-user signal separation takes place at the receiver side, using Successive Interference Cancellation (SIC) [2], [3].

The majority of existing works dealing with PD-NOMA have investigated the system-level performance in terms of system capacity and cell-edge user throughput [3]-[6]. They show that, by solving the user pairing, subband assignment and power allocation problems appropriately, PD-NOMA can provide a significant improvement compared to OMA.

In [2], system efficiency and user fairness of PD-NOMA are investigated and compared to OMA. In this sense, universal frequency reuse and proportional fairness (PF) scheduling [7] are adopted. A large throughput gain is observed for users located near the base station, but the gain achieved by celledge user throughput is shown to be rather limited.

Aiming at further enhancing user fairness and system capacity, we propose in this paper a resource allocation technique where fairness is targeted at each channel realization. By doing so, not only short-term fairness is achieved among users, but also system capacity and long-term fairness are consequently improved.

This paper is organized as follows: In Section II, we introduce the general system model and give a brief description of the NOMA-based PF scheduler. Section III details the proposed resource allocation schemes. Simulation results are given in Section IV, while Section V concludes the paper.

\section{SYSTEM DESCRIPTION}

\section{A. Description of the basic PD-NOMA scheme with SIC}

In this section, we describe the basic concept of PD-NOMA including user multiplexing at the transmitter of base station (BS) and signal separation at user terminal.

In this paper, a downlink air interface with a single transmitter and a single antenna receiver is considered. The system consists of $K$ users per cell, and a total bandwidth $B$ divided into $S$ subbands. Among the $K$ users, a set of users $U_{s}=$ $\left\{k_{1}, k_{2}, \ldots, k_{n}, \ldots, k_{n(s)}\right\}$ is selected to be scheduled on each frequency subband $s,(1 \leq s \leq S)$. The $n$-th $(1 \leq n \leq n(s))$ user scheduled at subband $s$ is denoted by $k_{n}$, and $n(s)$ indicates the number of users non-orthogonally scheduled at subband $s$. At the BS transmitter side, the information sequence of each scheduled user at subband $s$ is independently coded and modulated, resulting into symbol $x_{s, k_{n}}$ for the $n$-th scheduled user. Therefore, the signal $x_{s}$, transmitted by the 
BS on a certain subband $s$, represents the sum of the coded and modulated symbols of the $n(s)$ scheduled users:

$$
x_{s}=\sum_{n=1}^{n(s)} x_{s, k_{n}}, \text { with } \mathrm{E}\left[\left|x_{s, k_{n}}\right|^{2}\right]=P_{s, k_{n}}
$$

where $P_{s, k_{n}}$ is the power allocated to user $k_{n}$ at subband $s$. The received signal of user $k_{n}$ at subband $s, y_{s, k_{n}}$, is represented by:

$$
y_{s, k_{n}}=h_{s, k_{n}} x_{s, k_{n}}+w_{s, k_{n}}
$$

where $h_{s, k_{n}}$ is the channel coefficient between the BS and user $k_{n}$, at subband $s . w_{s, k_{n}}$ indicates the Gaussian noise plus inter-cell interference received by user $k_{n}$ on subband $s$. Let $P_{\max }$ be the BS maximum transmit power. Hence, the sum power constraint is formulated as:

$$
\sum_{s=1}^{S} \sum_{n=1}^{n(s)} P_{s, k_{n}}=P_{\max }
$$

At the receiver side, we consider SIC processing [8]. The optimal order for user decoding is in increasing order of the channel gains observed by the users, normalized by the noise and inter-cell interference $h_{s, k_{n}}^{2} / n_{s, k_{n}}$, where $h_{s, k_{n}}^{2}$ is the equivalent channel gain and $n_{s, k_{n}}$ the average power of $w_{s, k_{n}}$. Therefore, user $k_{n}$ can remove the inter-user interference from user $k_{j}$ at subband $s$, provided that $h_{s, k_{j}}^{2} / n_{s, k_{j}}$ is lower than $h_{s, k_{n}}^{2} / n_{s, k_{n}}$, and the received signals from other users with higher $h_{s, k_{j}}^{2} / n_{s, k_{j}}$ are treated as noise [9].

Assuming successful decoding and no error propagation, the throughput of user $k_{n}$, at subband $s, R_{s, k_{n}}$, is given by:

$R_{s, k_{n}}=\frac{B}{S} \log _{2}\left(1+\frac{h_{s, k_{n}}^{2} P_{s, k_{n}}}{\sum_{j \in \mathrm{U}_{s}, \frac{h_{s, k_{n}}^{2}}{n_{s, k_{n}}}<\frac{h_{s, k_{j}}^{2}}{n_{s, k_{j}}}}^{2} P_{s, k_{n}} P_{s, k_{j}}+n_{s, k_{n}}}\right)$

\section{B. Proportional fairness scheduler for PD-NOMA}

In [10], the operation of the PF scheduler is detailed: at the beginning of each scheduling slot, each user provides the base station with its channel state or its feasible rate. The PF algorithm keeps track of the historical rate $T_{k}(t)$, i.e., the average throughput of each user in a sliding window of length $t_{c}$. In scheduling slot $t$, a user is selected to be served based on:

$$
\arg \max _{k} \frac{R_{k}(t)}{T_{k}(t)}
$$

where $R_{k}(t)$ is the feasible rate of user $k$ in slot $t$.

An approximated version of the PF scheduler for multiple users transmission has been adopted in the majority of the works dealing with NOMA [9], [11], [12] for selecting users to be non-orthogonally scheduled on available resources. For each subband $s$, all possible candidate user sets are searched, and the set of scheduled users $U_{s}$ is chosen in such a way to maximize the PF scheduling metric :

$$
U_{s}=\underset{U}{\arg \max } \sum_{k \in U} \frac{R_{s, k \mid U}(t)}{T_{k}(t)}
$$

$U$ is a possible candidate user set, $R_{s, k \mid U}(t)$ is the instantaneous achievable throughput of user $k$ on subband $s$ at time instance $t$, the time index of a subframe. $R_{s, k \mid U}(t)$ is calculated based on Eq. 4, whereas $T_{k}(t)$ is updated according to:

$$
T_{k}(t+1)=\left(1-\frac{1}{t_{c}}\right) T_{k}(t)+\frac{1}{t_{c}} \sum_{s=1}^{S} R_{s, k}(t)
$$

The throughput averaging time window, $t_{c}$, is chosen such that a tradeoff between system performance in terms of fairness and system capacity is guaranteed.

\section{Proposed Resource Allocation Technique for A FAIR PD-NOMA SYSTEM}

The objective of the PF is to maximize the long-term averaged user rates, thus targeting long-term fairness. However, short-term fairness and fast convergence towards required performance are an important issue to be addressed in upcoming mobile standards [1]. Since the PF scheduler gives each user a priority inversely proportional to its average throughput, and since users instantaneous rates are not considered in the scheduling metric, it happens that some users do not gain access to available resources whenever their historical rates are high. This can be problematic for applications requiring a quasi-constant QoE, such as multimedia transmissions. In addition, the PF scheduler does not take advantage of the fact that the sum rate of NOMA systems increases when increasing the difference in channel gains of paired users. We propose a resource allocation technique that aims at addressing these issues.

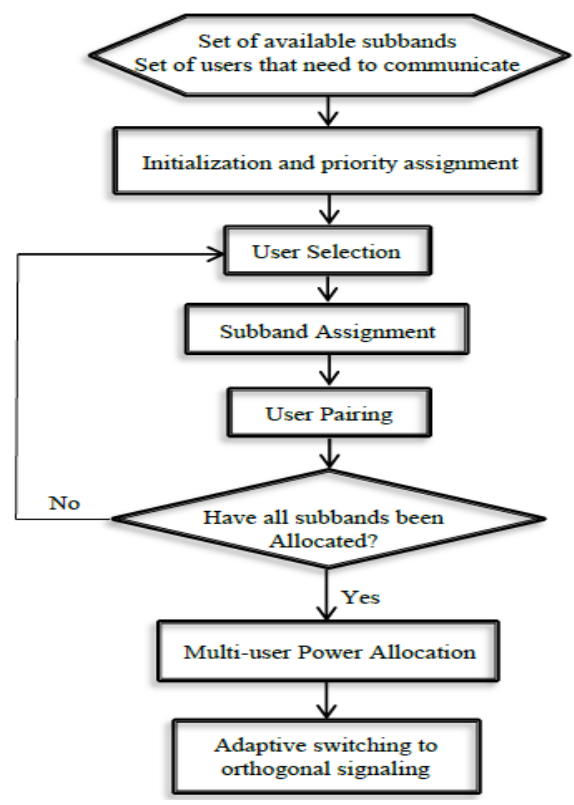

Fig. 1. Proposed resource allocation algorithm 
The design steps of the proposed resource allocation technique are depicted in Fig. 1 and detailed in the following subsections.

\section{A. Initialization and Priority Assignment}

At the beginning of the process, allocated powers and rates are all set to zero. Therefore, users are first chosen based on the priority constraint proposed in a previous work [6]. Priorities are assigned in the BS based on the channel coefficients experienced by users on available subbands. For each user $k$, its highest channel coefficient, $h_{s_{\max }, k}$, is selected among the channel gains experienced over all subbands. Then, the users are processed in the increasing order of $h_{s_{\max }, k}$ values: user $k_{\text {min }}$ with the lowest value of $h_{s_{\max }, k}$ is assigned first while user $k_{\max }$ with the highest value $h_{s_{\max }, k}$ is assigned last.

\section{B. User Selection and Subband Assignment}

After the initialization phase, unlike for PF scheduling, user rates already granted during the current allocation step are also taken into consideration. User $k_{1}$ is selected among the set of users that need to communicate as the user experiencing the lowest total achieved throughput. User selection can be written as follows:

$$
k_{1}=\min _{k}\left(\left(1-\frac{1}{t_{c}}\right) T_{k}(t)+\frac{1}{t_{c}} \sum_{s=1}^{S} R_{s, k}(t)\right)
$$

$R_{s, k}(t)$ is calculated based on Eq. 4 , and can be equal to zero if user $k$ is not scheduled on subband $s$.

User $k_{1}$ is then attributed the most favorable subband, i.e., the one with the highest channel coefficient, denoted by $s_{f}$.

\section{User Pairing}

We have limited our study to the case where the number of scheduled users per subband is equal to $2(n(s)=2)$, since it was shown in [13] that the improvement in cell throughput is $1 \%$ when the number of scheduled users per subband is equal to 3 , compared to the case where it is equal to 2 . However, the proposed pairing technique described in the following has the ability to consider more than 2 users per subband if needed.

Having determined the subband $s_{f}$ attributed to selected user $k_{1}$, the following step consists in selecting the best user $k_{2}$ to be paired with $k_{1}$. The selection of this second user is based on metric calculations requiring rate estimates, which in turn include power estimates. In the majority of papers dealing with PD-NOMA, such as [2], [12], an equal repartition of power among subbands is considered to simplify the scheduling problem. Quite the contrary, in our work, power is assigned to the users on the current subband $s_{f}$ based on the waterfilling principle, since it allows the achieved system throughput to be maximized [3]. The implementation of an optimal Power Allocation (PA) scheme based on a waterfilling process jointly performing intra and inter-subband PA is quite complex since it requires the resolution of a non-convex optimization problem. For this purpose, we have proposed a sub-optimal PA technique using an iterative waterfilling process [14], where only the user with the highest channel coefficient among scheduled users is considered in the inter-subband PA step. This technique was applied in the current work, combined with the Fractional Transmit Power Allocation (FTPA) technique [15] for intra-subband PA between the two paired users.

We have also introduced a new metric called Flexible Throughput vs. Fairness Maximization Metric (FTFMM) in order to cope with applications where throughput versus fairness maximization is essential. FTFMM aims at striking a flexible balancing between throughput and fairness, since it is based on the instantaneous rate on the considered subband $s_{f}$, the current user rates on the so far attributed subbands within the current allocation slot and on the historical rates of each possible candidate. A user is selected to be paired with user $k_{1}$ on the considered subband $s_{f}$ based on:

$$
k_{2}=\max _{k} \frac{R_{s_{f}, k}+R_{s_{f}, k_{1}}}{a R_{k, t o t}+b R_{k_{1}, t o t}}
$$

where $R_{s_{f}, k}$ is the data rate achieved by user $k$ over subband $s_{f}$, calculated from Eq. 4.

Parameters $a$ and $b$ can take values between 0 and 1 and are used to provide flexibility between throughput and fairness maximization. If $a$ is set to 1 and $b$ to 0 in Eq. 9, user $k_{2}$ is selected in a fair manner by taking the instantaneous rates as well as the total user rates of the possible candidates into consideration. On the contrary, if $a$ is set to 0 and $b$ to 1 , user $k_{2}$ is selected to be paired with $k_{1}$ according to its instantaneous rate achieved over $s_{f}$, and therefore the choice is only based on the rate maximization on the current subband. Neither his historical rate nor his current rate have been taken into account. In such a situation, the notion of fairness is completely flawed. Between these two extreme cases, and depending on the requirement of the application, parameters $a$ and $b$ can be varied in such a way to favor either the fairness or the throughput or to ensure balance between them.

\section{Adaptive switching to Orthogonal Signaling}

The estimation of power conducted within each stage of the allocation process is considered as temporary until the final stage is reached. At the final stage, once all subbands have been allocated, power is eventually distributed on each subband and data rates achieved by users are computed accordingly. However, the increase in spectral efficiency incurred by the use of NOMA compared to OMA is not systematic. Actually, the loss in data rate experienced by user $k_{1}$ when sharing its subband with user $k_{2}$ is sometimes significantly greater than the data rate gain achieved by $k_{2}$ on this subband. In this case, NOMA is not the appropriate solution, and the allocation of this subband to user $k_{1}$ alone turns out to be more beneficial. In a previous work [6], we have proposed to adaptively switch to orthogonal signaling if:

$$
\gamma\left(R_{s}-R_{s, k_{1}}\right)>R_{s, k_{2}}\left(\text { or } R_{s}>R_{s, k_{1}}+\frac{R_{s, k_{2}}}{\gamma}\right)
$$

where $R_{s}$ is the data rate achieved on subband $s$ by solely attributing the subband to a unique user. $\gamma$ is a control parameter to be determined a priori via simulations such as to maximize the system performance. 


\section{NUMERICAL RESUlTS}

\section{A. System Model Parameters}

Extensive system level simulations were carried out in order to evaluate the proposed allocation technique. The parameters considered in this work are based on existing LTE/LTE-Advanced specifications. The maximum BS transmission power is $46 \mathrm{dBm}$. The system bandwidth is $10 \mathrm{MHz}$ divided into $8,16,32,64$, or 128 subbands, with a carrier frequency of $2 \mathrm{GHz}$. The noise power spectral density is $4.10^{-18}$ $\mathrm{mW} / \mathrm{Hz}$. Users are randomly deployed in a cell of radius 500 meters, with a minimum distance of 35 meters between users and BS. Distance-dependent path loss is considered with a decay factor of 3.76. The Extended Typical Urban (ETU) channel model is used, with a mobile velocity of $50 \mathrm{~km} / \mathrm{h}$. We assume a $t_{c}$ window of $100 \mathrm{~ms}$ (100 subframes of $\left.1 \mathrm{~ms}\right)$. Perfect channel estimation is assumed. The maximum number of scheduled users per subband is $2(n(s)=2)$. The FTPA technique is applied for intra-subband PA with coefficient $\alpha$ equal to 0.7 [15].

\section{B. Performance Evaluation}

In this study, we mainly consider three system-level performance indicators: achieved system capacity, long-term fairness, and short-term fairness.

In order to evaluate the achieved fairness performance, we use the Gini fairness index [16], defined as follows:

$$
\begin{aligned}
G & =\frac{1}{2 K^{2} \bar{r}} \sum_{k^{\prime}=1}^{K} \sum_{k^{\prime \prime}=1}^{K}\left|r_{k^{\prime}}-r_{k^{\prime \prime}}\right| \\
\bar{r} & =\frac{\sum_{k=1}^{K} r_{k}}{K}
\end{aligned}
$$

Gini fairness index takes values between 0 and 1 , where $G=0$ corresponds to the maximum level of fairness among users, while a high value value of $G$ indicates that the resource allocation scenario is highly unfair. When fairness among users is to be evaluated within each scheduling slot, short-term fairness is considered and $r_{k}$ is equal to $R_{k}(t)$, the actual achieved throughput of user $k$ during scheduling slot $t$.

Fig. 2 and Fig. 3 compare the capacity of a NOMA system using the proposed approach with conventional OMA and NOMA systems based on PF scheduling, as a function of the number of users per cell and subbands, respectively. When different couples of parameters $a$ and $b$ values are evaluated, one can observe that the capacity is maximized for $a=0$ and $b=1$ (upper throughput bound), decreases progressively when $a$ increases and $b$ decreases, and reaches its lowest value for $a=1$ and $b=0$. This is due to the fact that when parameter $a$ is set to 0, Eq. 9 targets the maximization of the sum rates of paired users. As expected, a gain in performance is observed with the proposed FTFMM metric, when compared to a NOMA-based classical PF, since the proposed metric tries to provide rates to all users, even those experiencing bad channel conditions. For all the simulated systems, the throughput increases with the number of users per cell. This is

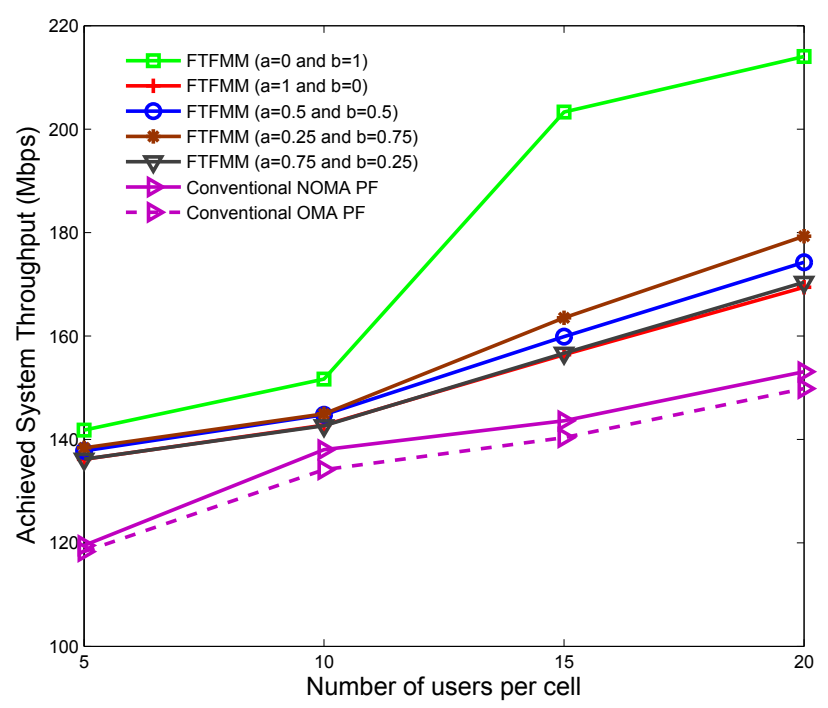

Fig. 2. Achieved system throughput of the proposed scheduling schemes, as a function of the number of users per cell

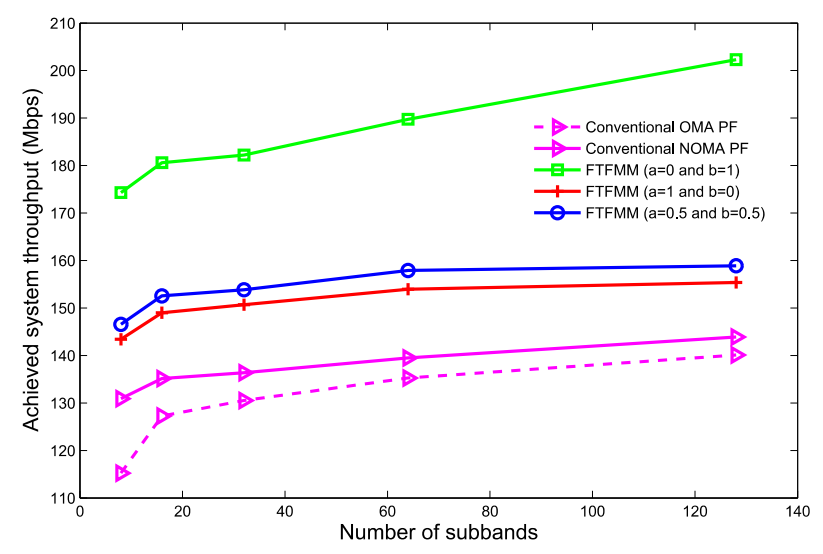

Fig. 3. Achieved system throughput of the proposed schemes, as a function of the number of subbands, for 15 users per cell

due to the fact that the higher the number of users per cell, the better the multi-user diversity is exploited by the scheduling scheme. For 15 users per cell, FTFMM with $a=0$ and $b=1$ allows the system throughput to be increased by $35 \%$ to $40 \%$ with respect to NOMA-based classical PF, depending on the number of subbands.

Fig. 4 and Fig. 5 compare the long-term fairness of a NOMA system using the proposed approach with conventional OMA and NOMA systems based on PF scheduling, as a function of the number of users per cell and subbands, respectively. One can observe that the highest level of fairness is achieved when $a=1$ and $b=0$ (upper fairness bound). FTFMM always shows better performance than the NOMA-based classical PF, whatever the number of users and subbands. Since it tends to achieve fairness in every scheduling slot by taking into account current rates, long-term fairness is enhanced consequently. 


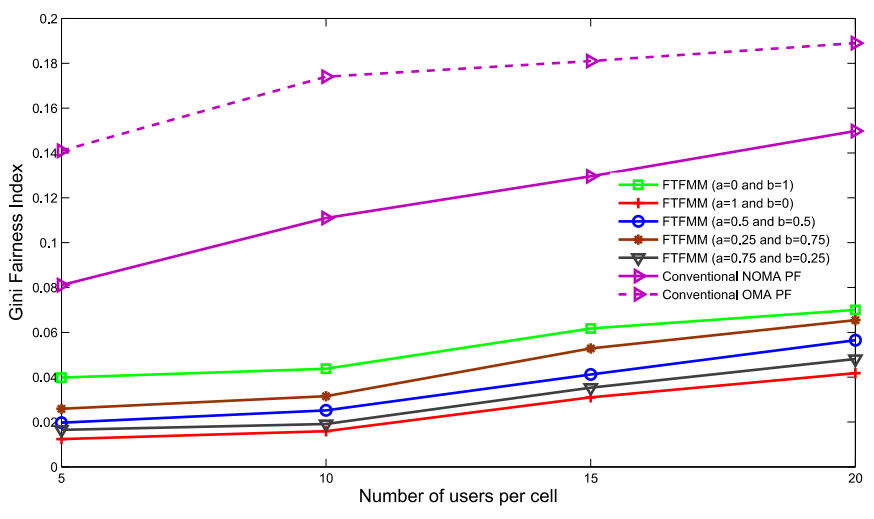

Fig. 4. Gini fairness index of the proposed scheduling schemes, as a function of the number of users per cell

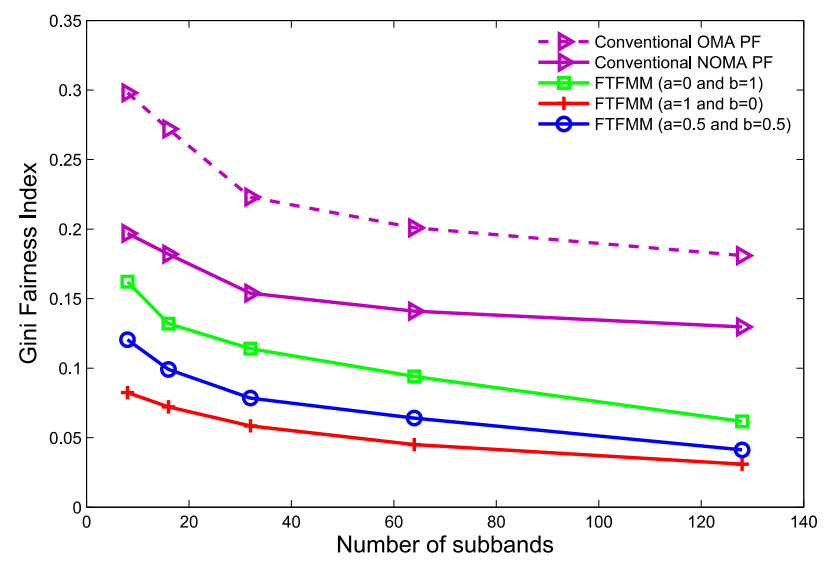

Fig. 5. Gini fairness index of the proposed schemes, as a function of the number of subbands, for 15 users per cell

This is in compliance with Eq. 9, where total achieved rates and current rates are both considered for user pairing, thus providing a high level of fairness.

Fig. 6 evaluates the evolution over time of the short-term fairness achieved by the proposed allocation schemes. The studied techniques are compared based on the time required to converge to a Gini fairness index close to zero. FTFMM always gives better fairness performance compared to classical $\mathrm{PF}$, for all simulated values of $a$ and $b$. Especially when $a=1$ and $b=0$, a high level of fairness is achieved in a limited number of allocation steps (less than 10 subframes for a Fairness index less than 0.1).

In order to assess the QoE achieved by users with the proposed scheduling schemes, we have also measured the rate latency, i.e., the waiting time before being served for the first time, and observed the variation of the user achieved rate versus the scheduling index $t$. For this purpose, Fig. 7 shows the achieved data rate of the user experiencing the largest rate latency versus time, for the different scheduling schemes under study. We can observe that no rate is provided to such users for the first five scheduling slots when classical PF is used. In addition, severe rate fluctuations are observed through

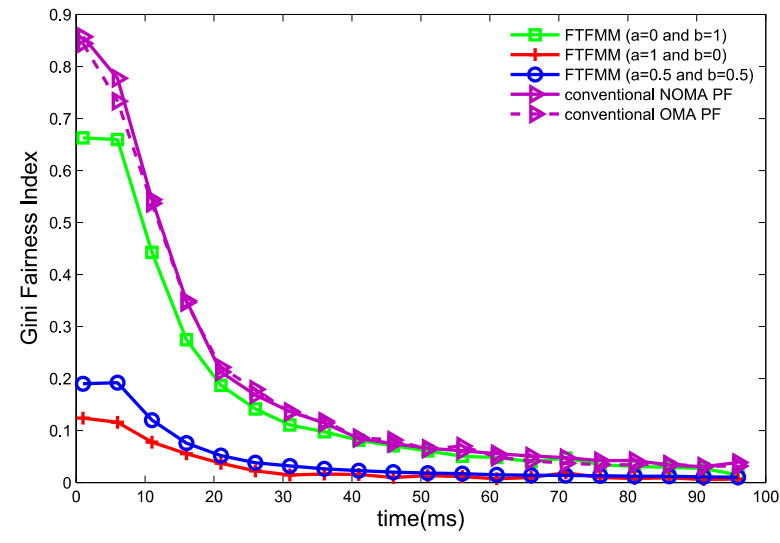

Fig. 6. Gini fairness index versus time for the proposed schemes

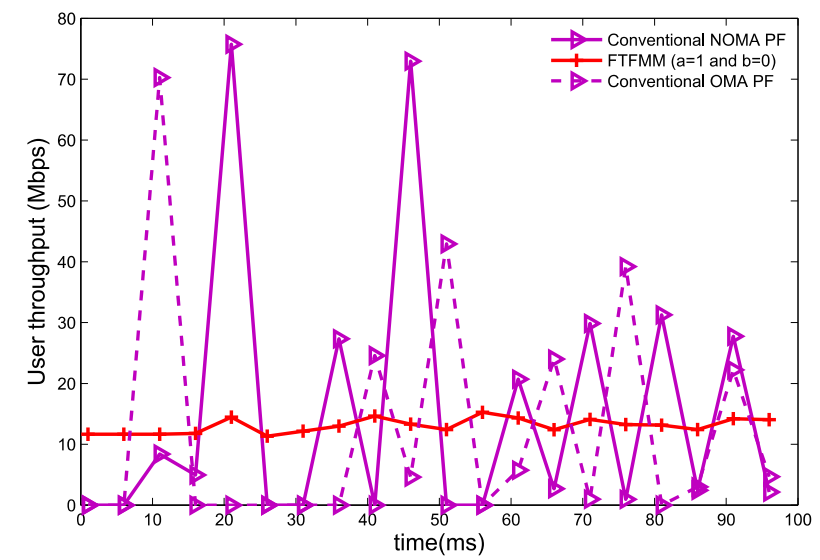

Fig. 7. User throughput versus time

time. On the contrary, with the proposed approach, a nonzero rate is allocated to the least privileged users from the first scheduling slot, and remains stable for all the following slots. With the conventional PF scheduler, users experiencing bad channel conditions are unlikely to be chosen and their achieved data rates is then equal to zero at the beginning of the scheduling process, while, using the proposed metric, zero rates are avoided thus suppressing the rate latency.

\section{CONCLUSION}

In this paper, we have proposed a novel resource allocation scheme for improving capacity and fairness of NOMA-based systems. For this purpose, a new pairing metric was designed, incorporating a non-uniform inter-subband power allocation scheme. This FTFMM metric targets a flexible balancing between throughput and fairness maximization. Simulation results show that the proposed scheme allow a significant increase in the system capacity and long-term fairness, when compared to a conventional NOMA-based PF scheduler. Furthermore, the proposed technique achieves a higher level of 
fairness within each scheduling slot, which improves the QoE of each user.

\section{ACKNOWLEDGMENT}

This work has been supported by the PHC CEDRE program.

\section{REFERENCES}

[1] http://www.ericsson.com/res/docs/2015/ericsson-mobility-report-june2015.pdf

[2] Otao, N., Kishiyama, Y., and Higuchi, K., "Performance of nonorthogonal access with SIC in cellular downlink using proportional fairbased resource allocation", in International Symposium on Wireless Communication Systems (ISWCS 2012), 2012.

[3] Saito, Y., Kishiyama, Y., Benjebbour, A., Nakamura, T., Li, A., and Higuchi, K., "Non-orthogonal multiple access (NOMA) for Cellular future radio access", in Proc. IEEE Vehicular Technology Conference (VTC 2013-Spring), 2013.

[4] Schaepperle, J., "Throughput of a wireless cell using superposition based multiple-access with optimized scheduling", in Proc. IEEE Personal Indoor and mobile radio Communications (PIMRC 2010), 2010.

[5] Ding, Z., Yang, Z., Fan, P., and Poor, H., "On the performance of non-orthogonal multiple access in $5 \mathrm{G}$ systems with randomly deployed users", IEEE Signal Processing Letters, 21(12),2014, pp. 1501-1505.

[6] Hojeij, M.R., Farah, J., AbdelNour, C., and Douillard, C., "New Optima and Suboptimal Resource Allocation Techniques for Downlink Nonorthogonal Multiple Access", Wireless Personal Communications, vol 87(3), 2016, pp. 837-867.

[7] Kelly, F.P., Maulloo, A.K., Tan, and D.K.H., "Rate control for communication networks: shadow prices, proportional fairness and stability", Journal of the Operational Research Society, vol 49(3), 1998, pp. 237252.

[8] Tse, D., and Viswanath, P., Fundamentals of Wireless Communication, Cambridge University Press, 2005.

[9] Tomida, S., and Higuchi, K., "Non-orthogonal Access with SIC in Cellular Downlink for User Fairness Enhancement", in Proc. International Symposium on Intelligent Signal Processing and Comm. Systems (ISPACS 2011), 2011.

[10] Viswanath, P., Tse, D., and Laroia, R., "Opportunistic Beamforming using Dumb Antennas", IEEE Transactions on Information Theory, vol 48(6), 2002, pp. 1277-1294.

[11] Saito, Y., Benjebbour, A., Kishiyama, Y, and Nakamura, T., "Systemlevel performance evaluation of downlink non-orthogonal multiple access (NOMA)", in Proc. IEEE International Symposium on Personal, Indoor and Mobile Radio Communications (PIMRC 2013), 2013.

[12] Benjebbour, A., Saito, Y., Kishiyama, Y., Li, A., Harada, A, and Nakamura, T., "Concept and practical considerations of non-orthogonal multiple access (NOMA) for future radio access", in Proc. International Symposium on Intelligent Signal Processing and Communication Systems (ISPACS), 2013.

[13] Y. Saito, Y., Benjebbour, A., Kishiyama,Y., and Nakamura, T.,"Systemlevel performance of downlink non-orthogonal multiple access (NOMA) under various environments" in proc. IEEE Vehicular Technology Conference (VTC 2015-Spring), 2015.

[14] Hojeij, M.-R., Abdel Nour, C., Farah, J., Douillard, C., "Waterfillingbased proportional fairness scheduler for downlink non-orthogonal multiple access", IEEE Wireless Communication Letters, 6(2), 2017, pp. 230-233.

[15] Benjebbour, A., Li, A., Saito, Y., Kishiyama, Y., Harada, A., and Nakamura, T., "System-level performance of downlink NOMA for future LTE enhancements" in Proc. IEEE Global Communications Conference (Globecom 2013), 2013.

[16] Dianati, M., Shen, X., and Naik, S., "A new fairness index for radio resource allocation in wireless networks", in Proc. IEEE Wireless Communication and Networking Conference, 2005. 